\title{
A PECUliar TRIANGULATION OF THE
}

\section{3-SPHERE 1}

\author{
A. ALTSHULER
}

\begin{abstract}
A triangulation of the 3-sphere with 10 vertices is presented, which is not directly obtainable by a generalized stellar subdivision from any 3 -sphere with 9 vertices. This answers in the affirmative a conjecture by B. Grünbaum. This example is shown to be the minimal possible.
\end{abstract}

1. Grünbaum's conjecture. The term combinatorial $n$-sphere is used here in its sense in [1] and [7], i.e., it is an abstract simplicial $n$-complex which, when geometrically realized (e.g. in the euclidean space $R^{2 n+1}$, see $[6$, p. 5]), is a triangulation of the topological $n$-sphere $S^{n}$. Thus, each combinatorial $n$ sphere $S$ has a certain number $f_{i}(S)$ of $i$-simplices for each $i, 0 \leqslant i \leqslant n$. Clearly $f_{0}(S) \geqslant n+2$. It is the purpose of the present work to present a combinatorial 3-sphere with 10 vertices which is not directly obtainable (defined below), thus solving in the affirmative a conjecture by Grünbaum [7, Conjecture 5].

Since all the complexes and spheres mentioned in the present work are combinatorial, we shall omit the adjective combinatorial. We use the terminology of [3] and [4], and bear in mind that an $n$-sphere is a particular case of a (combinatorial) $n$-manifold as defined there. The $n$-simplex whose vertices are $a_{0}, a_{1}, \ldots, a_{n}$ is denoted $a_{0} a_{1} \cdots a_{n}$.

A simple and natural way to obtain a 3 -sphere $S_{n+1}$ with $n+1$ vertices from a 3-sphere $S_{n}$ with $n$ vertices, is to apply on $S_{n}$ a generalized stellar subdivision, as defined in [7]. In this case we say that $S_{n+1}$ is directly obtainable from $S_{n}$. More precisely:

Following Alexander [1], we define an $m$-element to be any simplicial $m$ complex $C$ whose body is homeomorphic to a topological $n$-simplex $\Delta_{m}$. If $\phi$ is that homeomorphism, then the boundary bd $C$ of $C$ is the complex of all the simplices $\Delta \in C$ such that $\phi(\Delta)$ lies on the boundary of $\Delta_{m}$. It is clear that bd $C$ is independent of the particular homeomorphism $\phi$.

Let $S$ be an $m$-sphere with $n+1$ vertices $(m \geqslant 2, n \geqslant m+2)$. We would like to remove from $S$ one vertex $v \in S$ together with the complex $\operatorname{star}(v, S)$, and then complete the complex $S-\operatorname{star}(v, S)$ to an $m$-sphere $S^{\prime}$ with $n$ vertices. The "hole" created in $S$ by removing $\operatorname{star}(v, S)$ should be replaced by

Received by the editors January 27, 1975 and, in revised form, April 21, 1975.

AMS (MOS) subject classifications (1970). Primary 57A10, 55A99.

Key words and phrases. (Abstract) simplicial $n$-complex, combinatorial $n$-sphere, directly obtainable, shellable sphere.

1 This research was supported by a grant from the Israel National Commission for Basic Research. The author wishes to express his thanks to A. Bar-Iuda, who was responsible for the programing which led to the triangulation of the 3-sphere discussed in the present work. 
some $m$-element $C$ such that bd $C=\operatorname{link}(\nu, S)$, all the vertices of $C$ are in bd $C$, and $S^{\prime}=\operatorname{antistar}(v, S) \cup C$ is an $m$-sphere.

If this can be done, we say that $S$ can be refilled at the vertex $v$, and that $C$ is the refill. In this case, we also say that $S$ is directly obtainable at the vertex $v$ from $S^{\prime}$, since $S$ can be obtained from $S^{\prime}$ by replacing the subcomplex $C$ of $S^{\prime}$ by a new complex which has one new vertex $v$, and which will be $\operatorname{cl} \operatorname{star}(v, S)$. The last operation that yields $S$ from $S^{\prime}$ is the generalized stellar subdivision of $S^{\prime}$ mentioned in [7].

Notice that, as follows easily from [1, Theorem 12.5], if the $m$-sphere $S$ can be refilled at a vertex $v \in S$ and if $C$ is the refill, then $C \cap \operatorname{antistar}(v, S)$ $=\operatorname{link}(v, S)$, i.e., no interior simplex of $C$ is in $\operatorname{antistar}(v, S)$.

If $S$ and $S^{\prime}$ are $m$-spheres with $n+1$ and $n$ vertices, respectively, and there is a vertex in $S$ at which $S$ is directly obtainable from (an $m$-sphere combinatorially isomorphic to) $S^{\prime}$, we say that $S$ is directly obtainable from $S^{\prime}$. If $S$ is an $m$-sphere and there is some $m$-sphere $S^{\prime}$ from which $S$ is directly obtainable, we say that $S$ is directly obtainable. It is easy to see that every 2 sphere with $n>4$ vertices is directly obtainable.

The above mentioned conjecture by Grünbaum can now be stated as follows:

Grünbaum's Conjecture . For suitable $m \geqslant 3$ and $n \geqslant m+3$ there exists an $m$-sphere with $n$ vertices which is not directly obtainable.

2. A nondirectly obtainable 3-sphere. Let $S$ be the simplicial 3-complex with 10 vertices $0,1, \ldots, 9$ composed of the following 353 -simplices and their faces as shown in the list. We claim that $S$ is a 3 -sphere which is not directly obtainable.

\begin{tabular}{l|l|l|l|l}
0168 & 0246 & 1234 & 2456 & 1689 \\
0178 & 0359 & 1357 & 2567 & 1289 \\
0157 & 0349 & 1347 & 2579 & 3689 \\
0135 & 0459 & 1478 & 2679 & 3489 \\
0123 & 0456 & 1248 & 3679 & 2589 \\
0126 & 0567 & 3478 & 3678 & 2458 \\
0234 & 0678 & 3579 & 1269 & 4589
\end{tabular}

The first thing to notice is the fact that $S$ is neighborly, i.e., for every two vertices $x, y \in S$, the edge (1-simplex) $x y$ is also in $S$. Next notice that for every vertex $x \in S, \operatorname{link}(x, S)$ is a 2 -sphere, and therefore $S$ is a 3-manifold in the sense of [3]. Moreover, for every vertex $x \in S$, the 2 -sphere $\operatorname{link}(x, S)$ is combinatorially isomorphic to the 2-sphere shown in the Figure (while link $(0, S)$ is precisely the 2 -sphere in the Figure). The 2-sphere shown in the Figure is not stacked (see [3, Definition 2.1]; a stacked 2-sphere is essentially a dissection of a 3-ball as defined in [5]). Now, Theorem 2.4 of [3] formulated to suit our case states that if $M$ is a neighborly 3 -sphere directly obtainable at a vertex $x \in M$, then link $(x, M)$ is a stacked 2-sphere. Hence, assuming for the moment that $S$ is a 3 -sphere, we conclude that $S$ is not directly obtainable.

How can one prove that the 3 -manifold $S$ is indeed a 3 -sphere? It is easy to 
check that $S$ is connected and orientable. Since $\operatorname{star}(0, S)$ is a 1 -connected subcomplex of $S$ containing all the vertices of $S$, we can use Theorem 6.3.6 of [8] to compute the fundamental group (i.e., the first homotopy group) of $S$, and find that $S$ is simply connected. However, in the lack of a proof for the famous Poincaré Conjecture, it does not prove that $S$ is a 3-sphere.

The following construction yields that $S$ is indeed a 3-sphere. Start with the 3-element $\operatorname{star}(0, S)$, i.e., the complex composed of the first 143 -simplices in the list, and their faces. Its boundary is $\operatorname{link}(0, S)$ and is shown in the Figure. Add to it the remaining 213 -simplices of $S$, one at a time, according to their order in the list. In each step, except for the very last, we obtain a 3-element. The first step, e.g., which adds the 3-simplex 1234 to $\operatorname{star}(0, S)$, yields a 3element whose boundary is obtained from the Figure by removing the edge 23 and adding the edge 14 . The step before the last, adding the 3-simplex 2458, yields a 3 -element $C$ whose boundary is the boundary complex of the 3simplex 4589. The last step, which adds the 3-simplex 4589, closes $C$ to a $3-$ sphere (see [1, Theorem 14.1], also [10, Proposition 1.2]).

This construction yields also that the sphere $S$ is shellable, i.e., its 35 3simplices can be ordered $c_{1}, c_{2}, \ldots, c_{35}$ so that for each integer $k \leqslant 34$, $c_{1} \cup c_{2} \cup \cdots \cup c_{k}$ is a 3-element. The question whether or not there exists a nonshellable sphere-not to be confused with the question of the existence of a nonshellable cell, which is already solved in the affirmative-is still open, in spite of the contention of McMullen and Shephard [9, p. 177]. (A triangulated 3 -sphere $D$ is unshellable iff for every 3 -simplex $T$ in $D, D-T$ is an unshellable cell, see also [10].)

Since all the 3 -spheres with $n$ vertices $(5<n<10)$ are known to be directly obtainable (see [2], [3] and [4]), our 3-sphere $S$ is the "smallest" 3 sphere that is not directly obtainable.

The fact that all ten links link $(x, S)(x$ is a vertex in $S)$ are of the same type already indicates that $S$ possesses a high degree of symmetry. Indeed, the permutation $\psi=(0,2,8,3,5,6,1,4,9,7)$ of the ten vertices of $S$ induces a combinatorial automorphism of $S$, and using powers of $\psi$ it is clear that for every two vertices $x, y \in S$, there is a combinatorial automorphism of $S$ that maps $x$ to $y$.

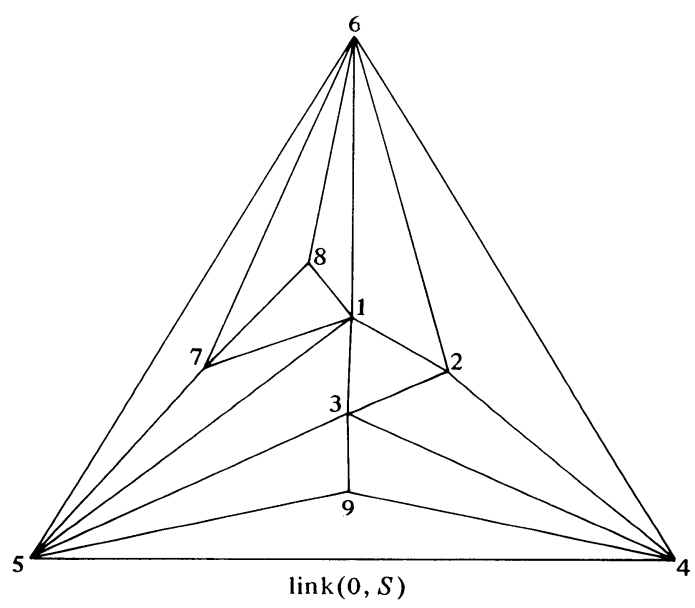

FIGURE 
For the sake of precision, we formalize our result as a theorem.

THEOREM. There exists a (triangulation of the) 3-sphere which is not directly obtainable. The minimum number of vertices in such a 3-sphere is 10. There exists a 3-sphere with 10 vertices which is neighborly, shellable, and not directly obtainable.

\section{REFERENCES}

1. J. W. Alexander, The combinatorial theory of complexes, Ann. of Math. 31 (1930), 292-320.

2. A. Altshuler, Combinatorial 3-manifolds with few vertices, J. Combinatorial Theory (A) 16 (1974), 165-173.

3. A. Altshuler and L. Steinberg, Neighborly combinatorial 3-manifolds with 9 vertices, Discrete Math. 8 (1974), 113-137.

4. - An enumeration of combinatorial 3-manifolds with 9 vertices, Discrete Math. (to appear).

5. L. W. Beineke and R. E. Pippert, The number of labelled dissections of a k-ball, Math. Ann. 191 (1971), 87-98. MR 44 \#94.

6. L. C. Glaser, Geometrical combinatorial topology. I, Van Nostrand Reinhold, New York, 1970.

7. B. Grünbaum, On the enumeration of convex polytopes and combinatorial spheres, ONR Technical Report, University of Washington, May 1969.

8. P. J. Hilton and S. Wylie, Homology theory: An introduction to algebraic topology, Cambridge Univ. Press, New York, 1960. MR 22 \#5663.

9. P. McMullen and G. C. Shephard, Convex polytopes and the upper bound conjecture, London Math. Soc. Lecture Note Series, 3, Cambridge Univ. Press, London and New York, 1971. MR 46 \#791.

10. G. Danaraj and V. Klee, Shellings of spheres and polytopes, Duke Math. J. 41 (1974), 443451 .

DFPARTMENT OF MATHEMATIC'S, BEN GURION UNIVERSITY OF THE NEGEV, BEER SHEVA,ISRAEL 